\title{
The Micro-mechanical Property of Porous Alumina Film on Glass Substrate Prepared by Oxalic Acid Oxidation
}

\author{
Pei-Tao GUO ${ }^{a *}$, Yu-Hong MAN ${ }^{b}$, Yong-Ping ZHANG \\ Faculty of Materials and Energy, Southwest University \\ aguopeitao@hotmail.com, bmanyh05@swu.edu.cn, czhangyyping@yahoo.com \\ ${ }^{*}$ Corresponding author
}

Keywords: Anodic Aluminum Oxide, Mechanical Property, Glass Substrate, Aluminum Foil.

\begin{abstract}
The mechanical properties of anodic aluminum oxide (AAO) on glass substrates prepared by $0.3 \mathrm{~mol} / \mathrm{L}$ oxalic acid solution are investigated by nanoindentation. The micro hardness, friction coefficient and Young's modulus are all associated with the porosity. The substrate is an important factor of the mechanical properties of AAO. The research shows that the mechanical property of AAO on glass substrates is a little higher than that of AAO on aluminum foil.
\end{abstract}

\section{Introduction}

AAO is considered to be an important material in nanotechnology fabrication, and is used as a template for the fabrication of other nanostructures such as nanotube and nanowire arrays, the porous structure of can be used in versatile applications such as a lubricant reservoir in self-lubricating systems [1], the friction coefficient reduced with the increase of load [2], and several studies have reported growth of AAO film by direct anodization of Al deposited on substrates such as silicon and glass [3-5]. The three-dimensionally nanostructured AAO films on glass substrate prepared in exhibited an angle-in sensitive optical transmission property [6]. Although the applications of AAO rely on its porous structure, processing, assemblage and exercising inevitably apply stress to this. Therefore, understanding the mechanical behavior is important to ensuring reliability and the usable range. In this paper, we have deposited aluminum on glass by heat evaporation and anodized aluminum into AAO film. The morphology and lattice structure have been characterized by scanning electron microscope (SEM). The mechanical properties of anodic aluminum oxide (AAO) on glass prepared by oxalic acids are investigated by nanoindentation.

\section{Experimental Details}

\section{Deposition of Aluminum Films}

Electron Beam Heating (EBH). Aluminum (purity>99.999\%) is coating material, and copper crucible the evaporation vessel, the aluminum thin films were deposited on glass by electron beam heating system in the ZZS700-6/G physical evaporation deposition system. The film thickness was monitored by the deposition time $(20 \mathrm{~s}, 60 \mathrm{~s})$. The base pressure in the deposition chamber was $2.7 \sim 3.0 \times 10^{-3} \mathrm{~Pa}$ and working pressure $6 \sim 3.5 \times 10^{-3} \mathrm{~Pa}$. The deposition beam current was $380 \mathrm{~mA}$. The source-substrate distance was $18 \mathrm{~cm}$ can make sure the better combined performance in both the uniformity in films thickness and the energy intensity of deposited particles.

Formation of Porous Alumina Films. Anodization is carried out in a $0.3 \mathrm{~mol} / \mathrm{L}$ oxalic acid solution at about $5^{\circ} \mathrm{C}$ with a $\mathrm{DC}$ constant oxidation voltage $\mathrm{V}_{\mathrm{a}}(20 \mathrm{~V}, 30 \mathrm{~V}, 40 \mathrm{~V})$. Because the formation of porous alumina is showed by a current recovery on its profile after a certain minimum, and the exhausting of porous alumina by a nearly zero current, the current profile is monitored to verify whether the alumina layer becomes porous or not and whether the alumina layer is exhausted or not. The aluminum films prepared in 2.1.1 and 2.1.2 were used as the anode and cleaned aluminum sheet as the cathode, the cathode is three times larger than the anode. The first anodization lasted $0.5 \mathrm{~h}$ and the second $1 \mathrm{~h}$. After the first anodization, the strip-off process was 
carried out in a mixture solution $\left(6 \% \mathrm{H}_{3} \mathrm{PO}_{4}+1.8 \% \mathrm{H}_{2} \mathrm{CrO}_{4}\right)$ at $60^{\circ} \mathrm{C}$ for $10 \mathrm{~min}$. The exposed and well-ordered concave patterns on the aluminum film acted as self-assembled mask for the second anodization process.

Characterization. The surface and the cross section of the films were examined with an FESEM (JSM-6700F). Crystal structures were examined by D/Max-IIIA X-Ray diffractometer with copper target.

The mechanical properties of thin films were performed by Nano indenter. The force, penetration and contact stiffness at $50 \mathrm{~Hz}$ during $250 \mathrm{~nm}$ deep indentation runs were recorded and converted to elastic modulus by the Oliver and Pharr method. The reduced elastic modulus Er of the film could be directly measured by the nanoindentation measurement. The elastic modulus of the film E could be calculated.

\section{Results and Discussion}

\section{Morphology Results of Porous Alumina Films}

Fig.1 shows the morphology different between AAO prepared by oxalic acid on glass substrate and aluminum foil. It is obvious that the pore in Fig. 1a is out of order and in Fig.1b is arranged in hexagonal. That's because the oxidation time of AAO on glass substrate is much less than on aluminum foil, so the pore without enough time to self-assemble to hexagonal arrangement. Because the thickness of AAO film is relate to the oxidation time, and so the thickness of AAO on glass substrate is much less than on aluminum foil.

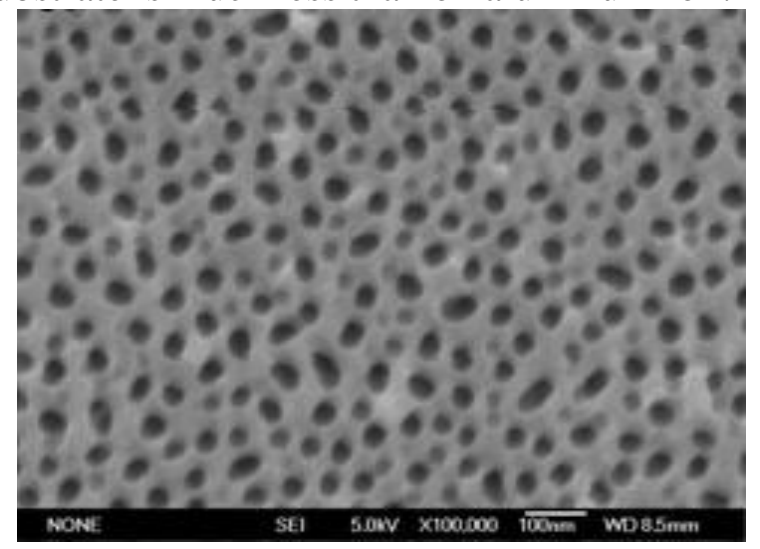

a:on glass

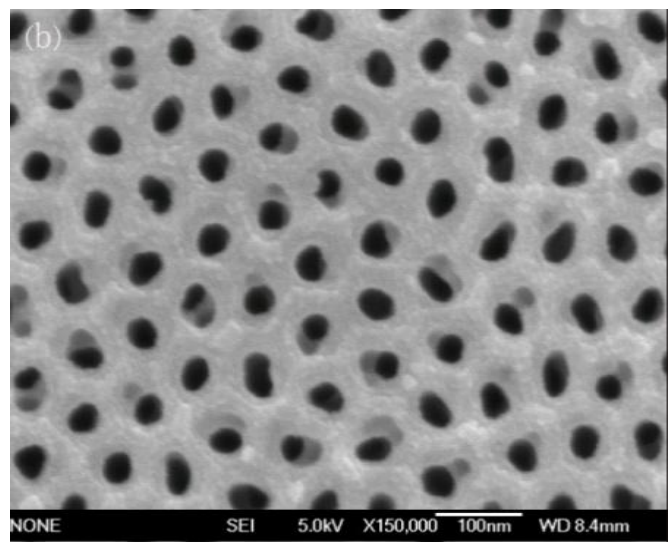

b: on aluminum foil

Fig.1 Morphology of AAO prepared by oxalic acid $(0.3 \mathrm{~mol} / \mathrm{L}, 30 \mathrm{~V})$

\section{Mechanical Properties of Porous Alumina Films}

It is shown in Fig. 2 that the average friction coefficient of AAO prepared by oxalic acid on glass under $20 \mathrm{~V}, 30 \mathrm{~V}, 40 \mathrm{~V}$, is $0.32,0.29$, and 0.31 respectively. The friction coefficient of these samples is all about 0.3 , the results can indeed be expected from the similar porosity percentages, which allow analogous amounts of debris entrapped in the pores and also result in similar penetration depths. The friction coefficient of AAO reported in paper [1] is 1.1 1.35 on aluminum foil, and much higher than us. That's because the AAO thickness on aluminum foil is thicker than on glass, and the friction coefficient of glass is lower than aluminum foil.

The micro-hardness of AAO as a function of indentation depth is shown in Fig.3a. It is obvious that the micro-hardness of AAO increased rapidly with the increase of indentation depth between $50-100 \mathrm{~nm}$ and increased slowly with the increase of indentation depth between 100-200nm. The micro-hardness of AAO as a function of load under different oxidation voltage is shown in Fig.3b. It is obvious that the micro-hardness of AAO decreased rapidly with the increase of different oxidation voltage from $20 \mathrm{~V}$ to $40 \mathrm{~V}$, and the micro-hardness test under $4000 \mu \mathrm{N}$ are little higher than test under $2000 \mu \mathrm{N}$. The test results are affected by the microstructure and the substrate, as Fig. 3 shows that the pore distribute on AAO on glass is smaller in diameter and the AAO film is thinner 
than that of AAO on aluminum foil, so the micro-hardness are effected by the glass substrate, when the indention depth and load increased the micro-hardness reflect apart of micro-hardness of glass.

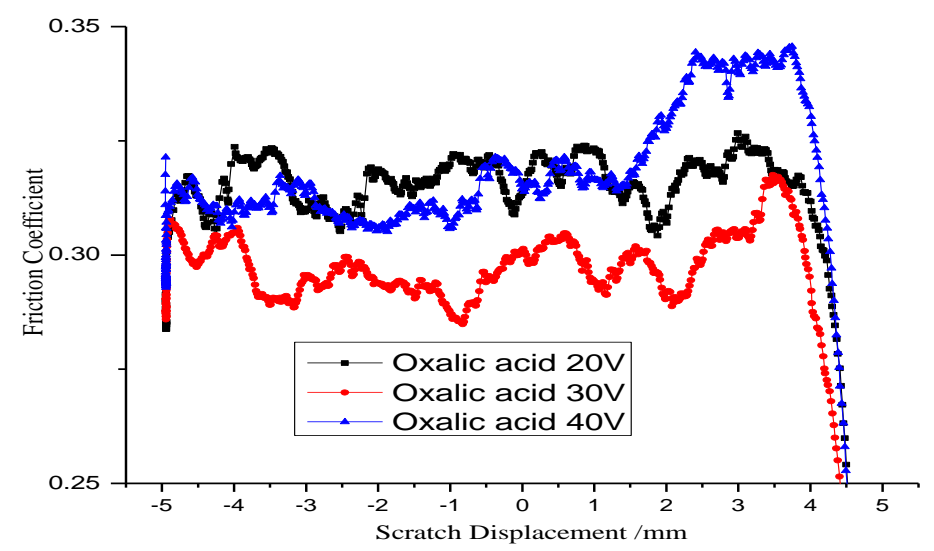

Fig. 2 Friction coefficient of AAO prepared by oxalic acid on glass $(4000 \mu \mathrm{N})$

a

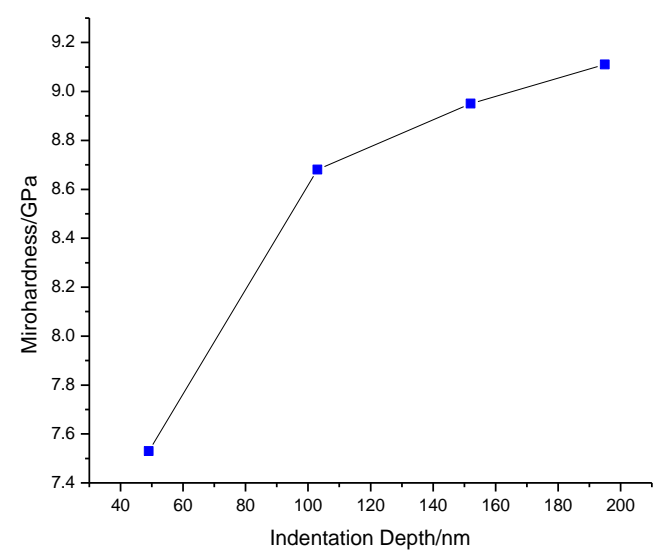

a: with different indentation depth b

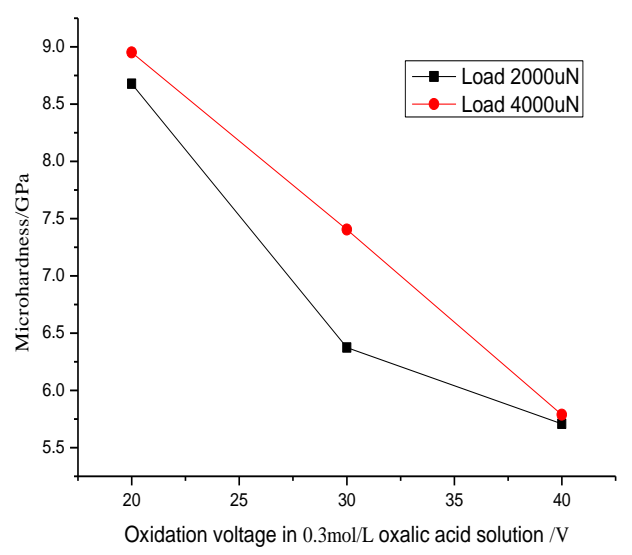

Fig.3 Microhardness of AAO prepared by oxalic acid on glass

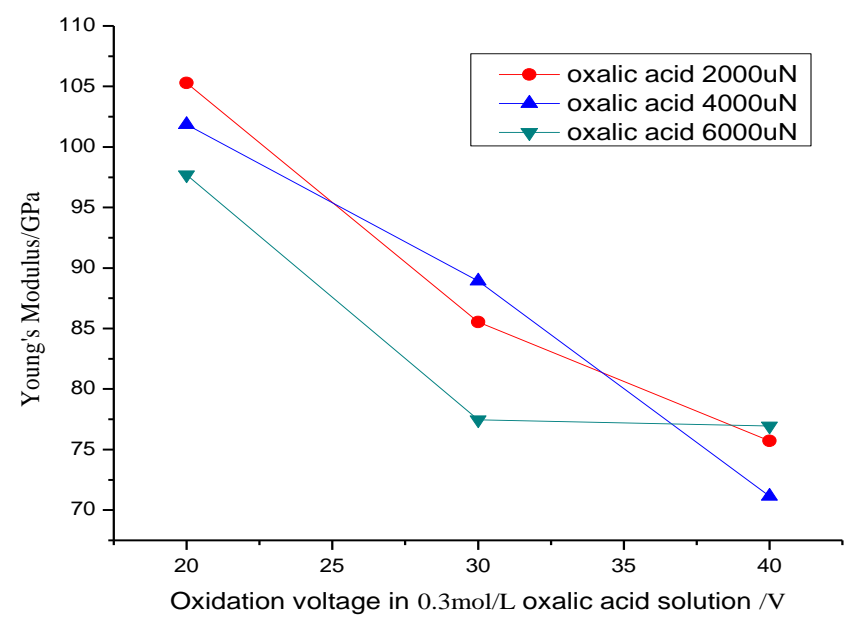

Fig.4 Young's modulus of AAO prepared by oxalic acid on glass with different load and oxidation voltage 
The Young's modulus of AAO as a function of indentation load and oxidation voltage is shown in Fig.4, the Young's modulus of AAO decreased with the increase of oxidation voltage. Indentation modulus of the AAO structure decreases monotically as the pore's size increases [7], and the pore concentration decreased rapidly with the increase of anodization voltage while the pore aperture is augments with the anodization voltage increasing [2]. There is a clear trend for Young's modulus to decrease with the increase of porosity level. The result reported in [8]. So it is reasonable that the Young's modulus is lower in $40 \mathrm{~V}$ oxidation voltage than $20 \mathrm{~V}$ oxidation voltage.

\section{Conclusions}

The micro hardness, friction coefficient and Young's modulus are all associated with the porosity and the substrate. The friction coefficients of AAO on glass substrate are all about 0.3 , and lower than on aluminum foil. The micro-hardness of AAO increased with the increase of indentation depth, decreased rapidly with the increase of different oxidation voltage from $20 \mathrm{~V}$ to $40 \mathrm{~V}$, and the micro-hardness test under $4000 \mu \mathrm{N}$ are little higher than test under $2000 \mu \mathrm{N}$. The Young's modulus $\mathrm{AAO}$ on glass substrate is lower in $40 \mathrm{~V}$ than $20 \mathrm{~V}$ oxidation voltage.

\section{Acknowledgements}

The author would like to express his gratitude to the Fundamental Research Funds for the Central Universities (XDJK2015C065) and Foundation of Southwest of University (SWU111055).

\section{References}

[1]Tsyntsaru, N., et al., Mechanical and frictional behaviour of nano-porous anodised aluminium. Materials Chemistry and Physics, 2014. 148(3): p. 887-895.

[2]Kim, H., et al., Tribological properties of nanoporous anodic aluminum oxide film. Surface and Coatings Technology, 2010. 205(5): p. 1431-1437.

[3]Ching-jung Yang, et al., fabrication of anodic aluminum oxide film on large-area glass substrate. electrochemical and solid-state letters, 2007. 10(12): p. C69-C71.

[4]Guo, P. and Z.L. Xia, Morphology and transmittance of porous alumina on glass substrate. applied surface science, 2011. 13(11): p. 7586-9.

[5]Peitao, G., et al., Effects of anodic oxidation process on transmittance of porous alumina on glass substrate. Advanced Materials Research, 2011. 179-180: p. 274-278.

[6]Katsuta, Y., et al., Three-dimensionally nanostructured alumina film on glass substrate: Anodization of glass surface. Journal of Non-Crystalline Solids, 2008. 354(2-9): p. 451-455.

[7]Ko, S., et al., Mechanical properties and residual stress in porous anodic alumina structures. Thin Solid Films, 2006. 515(4): p. 1932-1937.

[8]Jang, B. and H. Matsubara, Influence of porosity on hardness and Young's modulus of nanoporous EB-PVD TBCs by nanoindentation. Materials Letters, 2005. 59: p. 3462-3466. 